\title{
A Tourist-like MITE insertion in the upstream region of the BnFLC.A10 gene is associated with vernalization requirement in rapeseed (Brassica napus L.)
}

Jinna Hou', Yan Long ${ }^{1}$, Harsh Raman², Xiaoxiao Zou' ${ }^{1}$ Jing Wang ${ }^{1}$, Shutao Dai ${ }^{1}$, Qinqin Xiao ${ }^{1}$, Cong Li', Longjiang $\mathrm{Fan}^{3}$, Bin Liu ${ }^{4,5}$ and Jinling Meng ${ }^{1 *}$

\begin{abstract}
Background: Rapeseed (Brassica napus L.) has spring and winter genotypes adapted to different growing seasons. Winter genotypes do not flower before the onset of winter, thus leading to a longer vegetative growth period that promotes the accumulation and allocation of more resources to seed production. The development of winter genotypes enabled the rapeseed to spread rapidly from southern to northern Europe and other temperate regions of the world. The molecular basis underlying the evolutionary transition from spring- to winter- type rapeseed is not known, however, and needs to be elucidated.

Results: We fine-mapped the spring environment specific quantitative trait locus (QTL) for flowering time, qFT10-4, in a doubled haploid (DH) mapping population of rapeseed derived from a cross between Tapidor (winter-type) and Ningyou7 (semi-winter) and delimited the qFT10-4 to an 80-kb region on chromosome A10 of B. napus. The BnFLC.A10 gene, an ortholog of FLOWERING LOCUS C (FLC) in Arabidopsis, was cloned from the QTL. We identified 12 polymorphic sites between BnFLC.A10 parental alleles of the TN-DH population in the upstream region and in intron 1. Expression of both BnFLC.A10 alleles decreased during vernalization, but decreased more slowly in the winter parent Tapidor. Haplotyping and association analysis showed that one of the polymorphic sites upstream of BnFLC.A10 is strongly associated with the vernalization requirement of rapeseed $\left(r^{2}=0.93, x^{2}=0.50\right)$. This polymorphic site is derived from a Tourist-like miniature inverted-repeat transposable element (MITE) insertion/ deletion in the upstream region of BnFLC.A10. The MITE sequence was not present in the BnFLC.A10 gene in spring-type rapeseed, nor in ancestral ' $A$ ' genome species $B$. rapa genotypes. Our results suggest that the insertion may have occurred in winter rapeseed after $B$. napus speciation.

Conclusions: Our findings strongly suggest that (i) BnFLC.A10 is the gene underlying qFT10-4, the QTL for phenotypic diversity of flowering time in the TN-DH population, (ii) the allelic diversity caused by MITE insertion/ deletion upstream of BnFLC.A10 is one of the major causes of differentiation of winter and spring genotypes in rapeseed and (iii) winter rapeseed has evolved from spring genotypes through selection pressure at the BnFLC.A10 locus, enabling expanded cultivation of rapeseed along the route of Brassica domestication.
\end{abstract}

Keywords: Rapeseed, Flowering time, Vernalization, Tourist-like MITE, FLOWERING LOCUS C, Association analysis

\footnotetext{
* Correspondence: jmeng@mail.hzau.edu.cn

${ }^{1}$ National Key Laboratory of Crop Genetic Improvement, Huazhong

Agricultural University, Wuhan 430070, China

Full list of author information is available at the end of the article
} 


\section{Background}

Interaction between various environmental signals and flowering genes is critical for plants to flower and complete their life cycle, and thus important to humans, who rely upon adequate production of fruit and seeds to feed the world's growing population. Climate change fluctuations accompanying global warming $[1,2]$ are requiring plant breeders to elucidate the molecular mechanisms underlying flowering, and to develop strategies for manipulating and optimizing the flowering times to maximize crop yields. Four flowering pathways-autonomous, vernalization, photoperiod and gibberellic acid-have been established in Arabidopsis and partially identified in other species $[3,4]$. Vernalization is an adaptive trait in which plants acquire the ability to flower following exposure to cold temperatures. A series of genes in the endogenous network involved in this process, and their regulatory relationships, have been identified; genes from different flowering pathways function together with other integrator genes to control flowering $[5,6]$. The MADS-box family gene FLOWERING LOCUS C (FLC) represses flowering $[7,8]$ by suppressing the expression of FLOWERING LOCUS T $(F T)$, a key flowering integrator and confirmed florigen in plants [9-11], and other floral integrator genes such as FLOWERING DURATION and SUPPRESSOR OF OVEREXPRESSION OF CONSTANS [9,12,13]. Expression of $F L C$ is reduced by vernalization $[7,8] . F L C$ is upregulated by FRIGIDA (FRI) and repressed by genes in the autonomous pathway [14-16]. FLC expression has also been shown to be regulated via histone acetylation and methylation, which alters the expression of a trans-acting regulator common to FLC and members of the related MADS AFFECTING FLOWERING gene [17-20].

The genus Brassica, which diverged from Arabidopsis 14.5 to 20.4 million years ago [21-23] includes more crops of agricultural and horticultural importance than any other genus in the family of Brassicaceae. Comparative analysis has revealed that diploid Brassica genomes are composed of conserved segments triplicated from Arabidopsis $[24,25]$. The allopolyploid species B. napus (rapeseed, oilseed rape or canola; genomes AACC, $2 n=4 \mathrm{x}=$ 38 ) is a product of natural hybridization between diploid species $B$. rapa $(2 n=2 x=20$, genome $\mathrm{AA})$ and $B$. oleracea $(2 \mathrm{n}=2 \mathrm{x}=18$, genome $\mathrm{CC})$. Rapeseed originated in southern Europe along the coastline of the Mediterranean Sea $10,000-100,000$ years ago, and was domesticated as an oil crop 400-500 years ago. This crop was originally grown as a spring or semi-winter crop in Mediterranean climates; Its cultivation spread rapidly from southern into northern Europe after the development of winter rapeseed varieties, which do not flower during the long and cold winters. Understanding the evolution of flowering time is critical for domestication and introduction of rapeseed into new agroclimatic regions.
Miniature inverted-repeat transposable elements (MITEs) belong to a class of non-autonomous DNA transposable elements known as class II transposons. They are present in high copy number in the genome and contribute to genomic structure variations and intra-species diversity [26,27]. Differing MITE insertion profiles among varieties of a given species enable tolerance to environmental changes and allow adaptation under selective pressure [26,28,29].

Genetic analyses of several mapping populations of Brassica have revealed that both major and minor quantitative trait loci (QTLs) control flowering time. Some of these QTLs have also been shown to collocated with candidate genes for flowering time such as CO, FLC, FT and FRI [30-35]. Forty-two QTLs were identified in a doubled haploid $(\mathrm{DH})$ rapeseed mapping population (TN DH) derived from a cross between Tapidor and Ningyou7, but their magnitude and genetic effects varied with growing environment [36]. One major flowering time QTL, qFT10-4, which accounted for more than 50\% of phenotypic variation in flowering time in the TN DH populations grown in non-vernalization environments, was colocalized with the ortholog of $F L C$ from Arabidopsis in chromosome A10 and was designated BnFLC.A10 [36,37]. In our study, the candidate gene BnFLC.A10 for qFT10-4 was dissected using a map-based cloning approach, and an association was found between a Tourist-like MITE insertion/deletion in the upstream region of BnFLC.A10 and the stronger vernalization requirement in rapeseed.

\section{Results}

Cloning of BnFLC.A10 from qFT10-4 and allelic divergence

To construct a high-resolution map of the qFT10-4 locus, we analyzed a large $\mathrm{BC}_{5} \mathrm{~F}_{2}$ population $(9,000$ plants) that was derived from a cross between the DH line TN DH043 (winter-type) and Ningyou7 (semi-winter-type). Four molecular markers developed from the sequence of the Bacterial Artificial Chromosome (BAC) clone JBnB75D10, which contains BnFLC.A10, were used for the analysis (Figure 1A). Eight recombinants were identified and the QTL qFT10-4 was delimited to an 80-kb region that showed collinearity with the top of chromosome 5 of Arabidopsis thaliana (Figure 1B and C). None of the genes in this region except $F L C$ are known to be involved in floral transition.

To analyze the basis of the vernalization requirement in rapeseed, we cloned and compared BnFLC.A10 sequences (approximately $7 \mathrm{~kb}$ ) from Tapidor and Ningyou7, the parental lines of the mapping population. No polymorphism was found in the coding sequence (CDS) between the two alleles (BnFLC.A10-T and BnFLC.A10-N). However, there were two insertion/deletions (indels I and II) in the upstream region, together with two indels (indels III and IV) and eight single nucleotide polymorphisms (SNPs 1-8) in intron 1 of BnFLC.A10 (Figure 1D). Expression analysis 


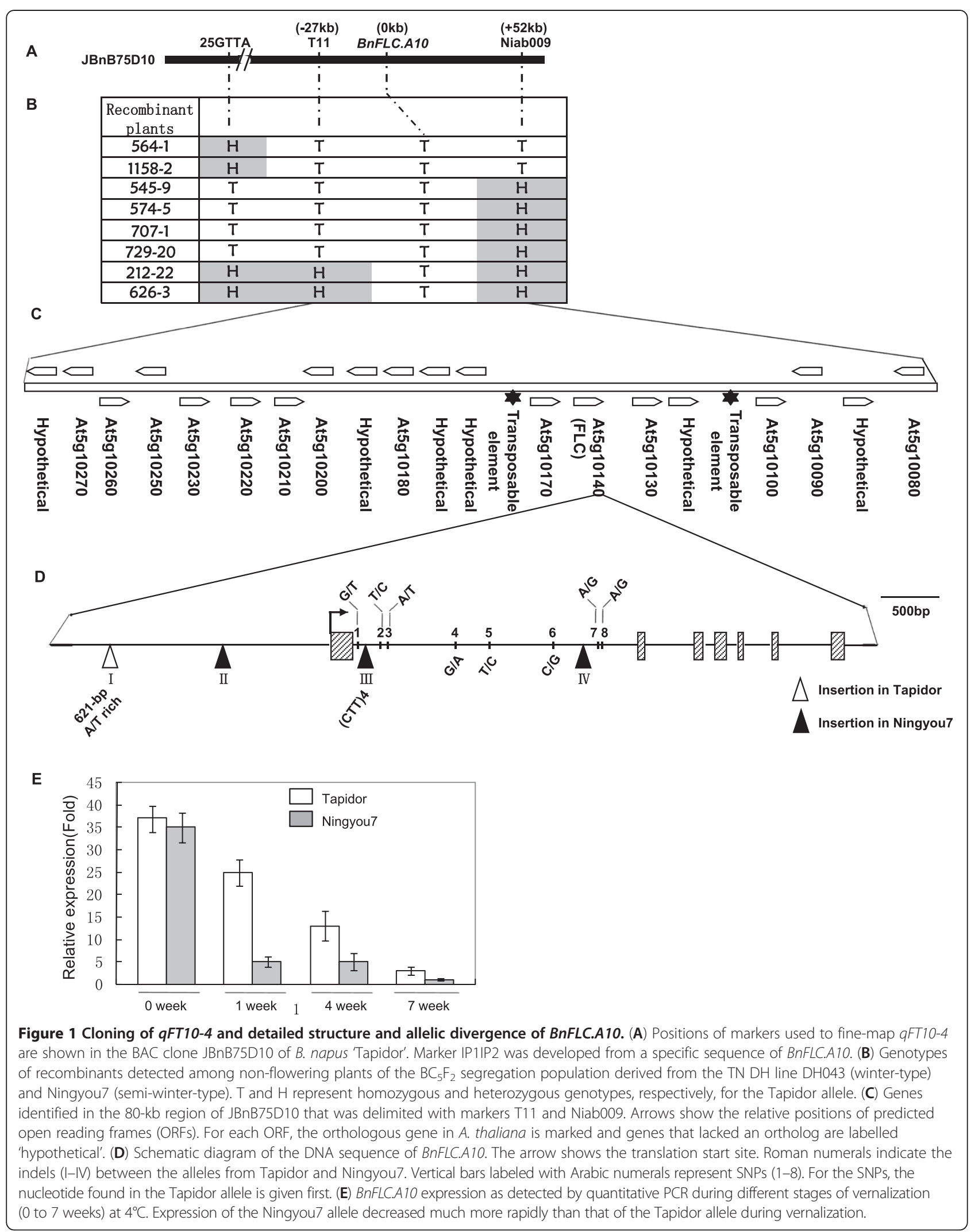


showed that BnFLC.A10-N was markedly down-regulated upon exposure to cold treatment after 1 week, whereas expression of BnFLC.A10-T decreased gradually over 7 weeks of cold treatment (Figure 1E). This observation provides strong evidence that BnFLC.A10 underlies variation for vernalization requirement and that differences in gene expression establish the basis for allelic variation at the qFT10-4 locus.

\section{A 621-bp insertion upstream of BnFLC.A10-T is associated with winter habit in rapeseed}

To determine whether sequence variations in the two BnFLC.A10 alleles contribute to differences in vernalization requirements or winter growth habit among natural rapeseed populations, we conducted an association analysis using a panel of 79 diverse rapeseed cultivars representing winter, semi-winter and spring genotypes. All of the cultivars were planted in spring environments. Because of lack of vernalization, none of the winter-type cultivars flowered; in contrast all of the spring-type and semi-winter-type cultivars (with one exception) flowered normally (Table 1). The three largest indels (I, II and IV) were analyzed first because the alleles could be easily distinguished by PCR (Figure 2A). For indel I, the 621-bp insertion was absent in all spring and semi-winter cultivars, but was present in all 18 winter cultivars except Coma. The 621-bp insertion showed a highly significant correlation with flowering phenotype $\left(r^{2}=0.93\right.$, Table 2; Figure $\left.2 B\right)$. In contrast, Indel II $\left(r^{2}=0.49\right)$ and indel IV $\left(r^{2}=0.56\right)$ were only weakly associated with flowering phenotype (Table 2).

Haplotyping of BnFLC.A10 specific markers for indels I-IV and SNPs 1-6 (Figure 1D) confirmed that most winter rapeseeds had a 621-bp insertion in the upstream region of BnFLC.A10 (haplotype I), whereas the 621-bp fragment was absent in the spring types (Table 2). These results suggest that indel I (with the 621-bp fragment present or absent) in the upstream region of BnFLC.A10 plays a very important role in modulating flowering time in natural rapeseed germplasm and potential development of a winter growth habit.

\section{The 621-bp insertion in the upstream region of BnFLC.A10} is a Tourist-like MITE

To further characterize the 621-bp insertion sequence in winter-type rapeseed accessions, BnFLC.A10 alleles from the eight cultivars that representing haplotype I (Table 2) were sequenced and aligned. All the sequenced genotypes showed 100\% identity. The inserted sequence possessed typical characteristics of a Tourist-like MITE [38-40], with 14-bp terminal inverted repeat (TIR) sequences flanked by target sequence duplications (TSDs) of TAA (Figure 3A). Between the TIR sequences, an AT-rich (67\%) core that contained 12 classes of important motifs (such as the TATA box and CAAT box) was identified (Additional file 1).
These motifs might function in transcriptional initiation or promotion, or in response to different stimuli and signals (Additional file 1). At least four homologs of the MITE insertion (BLASTN expected value $<1 \mathrm{e}^{-10}$ ) were identified in the genomic sequence of $B$. napus from public databases (http://www.ncbi.nlm.nih.gov) and up to 200 copies (E-value $<1 \mathrm{e}^{-10}$ ) were identified in the sequenced genome of $B$. rapa, the ancestral source of the A genome in $B$. napus. These homologs defined a new family of MITEs, which we named Monkey King (Figure 3B) after the subject of a Chinese myth. (In "Journey to the west", Monkey King is capable of 72 methods of transformation and can transform hundreds of monkeys with one of his hairs. He also jumps long distances with a cloud somersault).

\section{Origin and transmission of the BnFLC.A10 621-bp} insertion in $B$. napus and its $A$ genome ancestral species

\section{B. rapa}

To understand the evolutionary process behind the adaptation associated with the insertion of Monkey King into the upstream region of BnFLC.A10 and to trace its origin and transmission, we investigated an additional 154 spring cultivars of B. napus and 103 cultivars (including the genome sequenced cultivar, Chiifu [41]) belonging to nine subspecies of B. rapa (oilseed, swede and fodder types, Additional file 2). No Monkey King insertion was detected in the upstream region of BnFLC.A10 in any of the accessions, even though the empty site of insertion was almost $100 \%$ identical to the sequences that flanking the Monkey King insertion in BnFLC.A10 in winter rapeseed (Figure $3 \mathrm{~B}$ and Additional file 2). On the other hand, hundreds of copies of Monkey King were detected in the whole genome, but not in the BrFLC.A10 upstream region of B. rapa 'Chiifu'. This suggests that Monkey King may have pre-existed in the $B$. rapa genome but was inactive, after the generation of $B$. napus, it was activated and inserted into the upstream region of BnFLC.A10, giving rise to winter rapeseed.

\section{Discussion}

In this study, we used positional cloning to dissect the major flowering time QTL, qFT10-4, which was detected only in the spring-cropped TN DH population. The QTL qFT10-4 on chromosome A10 was delimited in a narrow 80-kb genomic region and annotation of different genes allowed us to identify BnFLC.A10, an ortholog of FLC, as the candidate gene. We demonstrated for the first time that flowering time variation at the qFT.10-4 locus is conditioned by the major vernalization response gene, $B n F L C$. A10; the MITE insertion upstream of BnFLC.A10 show significant association with the flowering time variation between winter and spring rapeseed.

Control of flowering time by vernalization has previously been shown to depend on a complex regulatory network, especially in amphidiploid rapeseed. In one study of 
Table 1 Phenotypic and genotypic data for 79 B. napus accessions

\begin{tabular}{|c|c|c|c|c|c|c|c|c|}
\hline \multirow[t]{2}{*}{ Accession } & \multirow[t]{2}{*}{ Origin } & \multirow[t]{2}{*}{ Type } & \multicolumn{3}{|c|}{ Genotype $^{a}$} & \multicolumn{3}{|c|}{ Days to flowering } \\
\hline & & & InDel I & InDel II & InDel IV & 2007 & 2008 & 2009 \\
\hline Altex & Canada & \multirow[t]{30}{*}{ Spring } & $\mathrm{N}$ & $\mathrm{T}$ & $T$ & 69 & 61 & 64 \\
\hline Alto & Canada & & $\mathrm{N}$ & $\mathrm{T}$ & $\mathrm{T}$ & 65 & 61 & 61 \\
\hline Apomix & Unknown & & $\mathrm{N}$ & $\mathrm{N}$ & $\mathrm{N}$ & 65 & 61 & 61 \\
\hline Bronowski DH2 & Poland & & $\mathrm{N}$ & $\mathrm{N}$ & $\mathrm{N}$ & 89 & 85 & 87 \\
\hline Bullet & Canada & & $\mathrm{N}$ & $\mathrm{T}$ & $\mathrm{T}$ & 67 & 61 & 63 \\
\hline Celebra & Unknown & & $\mathrm{N}$ & $\mathrm{N}$ & $\mathrm{N}$ & 72 & 72 & 75 \\
\hline CENN & Unknown & & $\mathrm{N}$ & $\mathrm{N}$ & $\mathrm{N}$ & 78 & 73 & 77 \\
\hline Comet & Denmark & & $\mathrm{N}$ & $\mathrm{T}$ & $\mathrm{T}$ & 75 & 68 & 68 \\
\hline Conzuul & Unknown & & $\mathrm{N}$ & $\mathrm{T}$ & - & 74 & 68 & 69 \\
\hline D.ARoll & Unknown & & $\mathrm{N}$ & $\mathrm{T}$ & $\bar{T}$ & 72 & 67 & 67 \\
\hline Dac-chosen & Unknown & & $\mathrm{N}$ & $\mathrm{N}$ & $\mathrm{N}$ & 67 & 67 & 67 \\
\hline Dunkeld & Australia & & $\mathrm{N}$ & $N$ & $N$ & 71 & 71 & 73 \\
\hline Erglu & Germany & & $\mathrm{N}$ & $\mathrm{N}$ & $\mathrm{N}$ & 76 & 64 & 66 \\
\hline Erra & Germany & & $\mathrm{N}$ & $N$ & $\mathrm{~N}$ & 69 & 70 & 72 \\
\hline Global & Canada & & $\mathrm{N}$ & $\mathrm{T}$ & $\mathrm{T}$ & 78 & 64 & 66 \\
\hline Granit & Sweden & & $\mathrm{N}$ & $\mathrm{T}$ & $T$ & 79 & 59 & 62 \\
\hline Grouse & Australia & & $\mathrm{N}$ & $\mathrm{T}$ & - & 69 & 60 & 57 \\
\hline GULLR & Sweden & & $\mathrm{N}$ & $\mathrm{T}$ & $\mathrm{T}$ & 78 & 73 & 73 \\
\hline Jiayou1 & Canada & & $\mathrm{N}$ & $N$ & $N$ & 76 & 91 & 78 \\
\hline Jiayou3 & Canada & & $\mathrm{N}$ & $\mathrm{N}$ & $\mathrm{N}$ & 75 & 77 & 74 \\
\hline Karoo & Australia & & $\mathrm{N}$ & $\mathrm{N}$ & $\mathrm{N}$ & 65 & 58 & 66 \\
\hline Marnoo & Australia & & $\mathrm{N}$ & $\mathrm{N}$ & $\mathrm{N}$ & 69 & 65 & 67 \\
\hline Monty & Australia & & $\mathrm{N}$ & $\mathrm{T}$ & $T$ & 69 & 56 & 60 \\
\hline Niklas & Sweden & & $\mathrm{N}$ & $\mathrm{T}$ & $\mathrm{T}$ & 78 & 67 & 72 \\
\hline Nilla & Sweden & & $\mathrm{N}$ & $\mathrm{T}$ & $\mathrm{T}$ & 82 & 77 & 73 \\
\hline Ning RS-1 & China & & $\mathrm{N}$ & $\mathrm{N}$ & - & 83 & 79 & 81 \\
\hline Qingyou2 & China & & $\mathrm{N}$ & $\mathrm{N}$ & $\mathrm{N}$ & 65 & 60 & 63 \\
\hline Rioklas & Unknown & & $\mathrm{N}$ & $\mathrm{T}$ & $\mathrm{T}$ & 75 & 73 & 74 \\
\hline Rucabo & Germany & & $\mathrm{N}$ & $\mathrm{N}$ & $\mathrm{N}$ & 74 & 68 & 69 \\
\hline Westar & Canada & & $\mathrm{N}$ & $\mathrm{T}$ & $\mathrm{T}$ & 50 & 50 & 50 \\
\hline Chuanyou11 & China & \multirow[t]{16}{*}{ Semi-winter } & $\mathrm{N}$ & $\mathrm{N}$ & $N$ & 67 & 71 & 72 \\
\hline Fuyou1 & China & & $\mathrm{N}$ & $\mathrm{N}$ & $\mathrm{N}$ & 66 & 69 & 67 \\
\hline Fuyou2 & China & & $\mathrm{N}$ & $\mathrm{T}$ & $\mathrm{H}$ & 67 & 65 & 65 \\
\hline Gànyou14 & China & & $\mathrm{N}$ & $\mathrm{T}$ & $\mathrm{H}$ & 75 & 77 & 76 \\
\hline Gānyou2 & China & & $\mathrm{N}$ & $\mathrm{N}$ & $\mathrm{N}$ & 67 & 77 & 77 \\
\hline Gànyou3 & China & & $\mathrm{N}$ & $N$ & $N$ & 69 & 67 & 68 \\
\hline Gānyou5 & China & & $\mathrm{N}$ & $\mathrm{N}$ & $\mathrm{N}$ & 67 & 62 & 63 \\
\hline Huashuang1 & China & & $\mathrm{N}$ & $\mathrm{N}$ & $\mathrm{N}$ & 72 & 72 & 73 \\
\hline Huashuang2 & China & & $\mathrm{N}$ & $\mathrm{N}$ & $\mathrm{T}$ & 81 & 82 & 78 \\
\hline Huashuang3 & China & & $\mathrm{N}$ & $\mathrm{T}$ & $\mathrm{T}$ & 75 & 72 & 68 \\
\hline Huáyou10 & China & & $\mathrm{N}$ & $N$ & $\mathrm{~N}$ & 69 & 69 & 69 \\
\hline Huáyou11 & China & & $\mathrm{N}$ & $\mathrm{N}$ & $\mathrm{N}$ & 68 & 68 & 68 \\
\hline Huáyou12 & China & & $\mathrm{N}$ & $N$ & $\mathrm{~N}$ & 69 & 69 & 69 \\
\hline Huáyou13 & China & & $\mathrm{N}$ & $\mathrm{N}$ & $\mathrm{N}$ & 72 & 72 & 72 \\
\hline Huáyou14 & China & & $\mathrm{N}$ & $\mathrm{N}$ & $\mathrm{N}$ & 71 & 71 & 71 \\
\hline Huáyou16 & China & & $\mathrm{N}$ & $\mathrm{N}$ & - & 72 & 76 & 79 \\
\hline
\end{tabular}


Table 1 Phenotypic and genotypic data for 79 B. napus accessions (Continued)

\begin{tabular}{|c|c|c|c|c|c|c|c|c|}
\hline Huáyou2 & China & & $\mathrm{N}$ & $\mathrm{T}$ & $\mathrm{N}$ & 67 & 67 & 73 \\
\hline Huāyou3 & China & & $\mathrm{N}$ & $\mathrm{N}$ & $\mathrm{N}$ & 74 & 74 & 74 \\
\hline Huāyou4 & China & & $\mathrm{N}$ & $\mathrm{N}$ & $\mathrm{N}$ & 63 & 63 & 63 \\
\hline Huāyou6 & China & & $\mathrm{N}$ & $\mathrm{N}$ & $\mathrm{N}$ & 67 & 67 & 67 \\
\hline Huāyou9 & China & & $\mathrm{N}$ & $\mathrm{N}$ & $\mathrm{N}$ & $\mathrm{NF}$ & NF & $\mathrm{NF}$ \\
\hline huāyuo5 & China & & $\mathrm{N}$ & $\mathrm{T}$ & $\mathrm{T}$ & 69 & 68 & 62 \\
\hline Huáyuo6 & China & & $\mathrm{N}$ & $\mathrm{T}$ & - & 61 & 68 & 72 \\
\hline Suyou3 & China & & $\mathrm{N}$ & $\mathrm{N}$ & $\mathrm{N}$ & 62 & 59 & 59 \\
\hline Xiangnongyou2 & China & & $\mathrm{N}$ & $\mathrm{N}$ & $\mathrm{N}$ & 70 & 70 & 70 \\
\hline Xiangnongyou3 & China & & $\mathrm{N}$ & $N$ & $\mathrm{~N}$ & 67 & 67 & 67 \\
\hline Xiangyou13 & China & & $\mathrm{N}$ & $\mathrm{N}$ & $\mathrm{N}$ & 70 & 73 & 74 \\
\hline Youyan2 & China & & $\mathrm{N}$ & $\mathrm{N}$ & $\mathrm{N}$ & 64 & 60 & 61 \\
\hline Zhenyou-1 & China & & $\mathrm{N}$ & $\mathrm{T}$ & $\mathrm{H}$ & 67 & 78 & 80 \\
\hline Zheyou7 & China & & $\mathrm{N}$ & $\mathrm{N}$ & $\mathrm{N}$ & 72 & 67 & 69 \\
\hline Apache & UK & Winter & $\mathrm{T}$ & $\mathrm{T}$ & $\mathrm{T}$ & $\mathrm{NF}$ & NF & NF \\
\hline Bakow & Poland & & $\mathrm{T}$ & $\mathrm{T}$ & $\mathrm{T}$ & $\mathrm{NF}$ & $N F$ & NF \\
\hline Bienvenu & France & & $\mathrm{T}$ & $\mathrm{T}$ & $\mathrm{T}$ & $\mathrm{NF}$ & NF & NF \\
\hline Bolko & Poland & & $\mathrm{T}$ & $\mathrm{T}$ & $\mathrm{T}$ & $\mathrm{NF}$ & NF & $\mathrm{NF}$ \\
\hline Brutor & France & & $\mathrm{T}$ & $\mathrm{T}$ & $\mathrm{T}$ & $\mathrm{NF}$ & NF & NF \\
\hline Casino & Sweden & & $\mathrm{T}$ & $\mathrm{T}$ & $\mathrm{T}$ & $\mathrm{NF}$ & NF & $\mathrm{NF}$ \\
\hline Ceres & Germany & & $\mathrm{T}$ & $\mathrm{T}$ & $\mathrm{T}$ & $\mathrm{NF}$ & NF & $\mathrm{NF}$ \\
\hline Coma & Unknown & & $\mathrm{N}$ & $\mathrm{T}$ & $\mathrm{H}$ & $\mathrm{NF}$ & NF & $\mathrm{NF}$ \\
\hline D-083 & Unknown & & $\mathrm{T}$ & $\mathrm{T}$ & $\mathrm{T}$ & $\mathrm{NF}$ & NF & NF \\
\hline Diadem & Germany & & $\mathrm{T}$ & $\mathrm{T}$ & $\mathrm{T}$ & $\mathrm{NF}$ & NF & NF \\
\hline JeT-Neuf & France & & $\mathrm{T}$ & $\mathrm{T}$ & $\mathrm{T}$ & $\mathrm{NF}$ & NF & NF \\
\hline Jupiter & Sweden & & $\mathrm{T}$ & $\mathrm{T}$ & $\mathrm{T}$ & $\mathrm{NF}$ & NF & NF \\
\hline Libritta & Germany & & $\mathrm{T}$ & $\mathrm{T}$ & $\mathrm{T}$ & $\mathrm{NF}$ & NF & NF \\
\hline Liradonna & Germany & & $\mathrm{T}$ & $\mathrm{T}$ & $\mathrm{T}$ & NF & NF & NF \\
\hline Literavo & Germany & & $\mathrm{T}$ & $\mathrm{T}$ & $\mathrm{T}$ & $\mathrm{NF}$ & NF & NF \\
\hline Matador & Sweden & & $\mathrm{T}$ & $\mathrm{T}$ & $\mathrm{T}$ & $\mathrm{NF}$ & NF & $\mathrm{NF}$ \\
\hline Nestor & Sweden & & $\mathrm{T}$ & $\mathrm{T}$ & $\mathrm{T}$ & $\mathrm{NF}$ & NF & $\mathrm{NF}$ \\
\hline Panter & Sweden & & $\mathrm{T}$ & - & $\mathrm{T}$ & $\mathrm{NF}$ & NF & $\mathrm{NF}$ \\
\hline Quinta & Germany & & $\mathrm{T}$ & $\mathrm{T}$ & $\mathrm{T}$ & $\mathrm{NF}$ & NF & NF \\
\hline
\end{tabular}

${ }^{\mathrm{a}} \mathrm{T}, \mathrm{N}$ and $\mathrm{H}$ indicate homozygous for Tapidor, homozygous for Ningyou7 and heterozygous genotypes, respectively; - represents an undetectable genotype or a new genotype distinct from the two parents.

The gene.

the relationship between flowering time and FLC orthologs, five $B n F L C$ sequences were isolated from $B$. napus cDNA library and in another study six FLC paralogs have been identified in $B$. napus by comparative analysis of $B$. napus and Arabidopsis genomes [36,42]. The fact that indel I in the upstream region of BnFLC.A10 cosegregated with flowering phenotype in the TN DH population but in only some of the diverse cultivars might be due to the contribution of other flowering time QTLs, including other BnFLCs, with very small genetic effects. For examples, one of the BnFLC paralogs, which was located in linkage group A3 (BnFLC.A3b), colocalized with the flowering time QTL and thus might contribute to the vernalization response in certain cultivars [43]. In fact, at least nine copies of Bn.FLC genes exist in rapeseed [43]. Other genes, such as FRIGIDA, also regulate FLC expression in rapeseed; BnaA.FRI.a, one of orthologs of FRIGIDA in Arabidopsis, contributes to flowering time variation in rapeseed, which may partly explain why indel I did not totally cosegregated with flowering time in the association analysis [33]. In our research, the cultivar "Coma" that lacked the BnFLC.A10 upstream MITE insertion still exhibited the winter characteristic. It is thus possible that other copies of BnFLCs or related genes from the vernalization pathway may contribute to vernalization response in $B$. napus. The expression of one of the other 


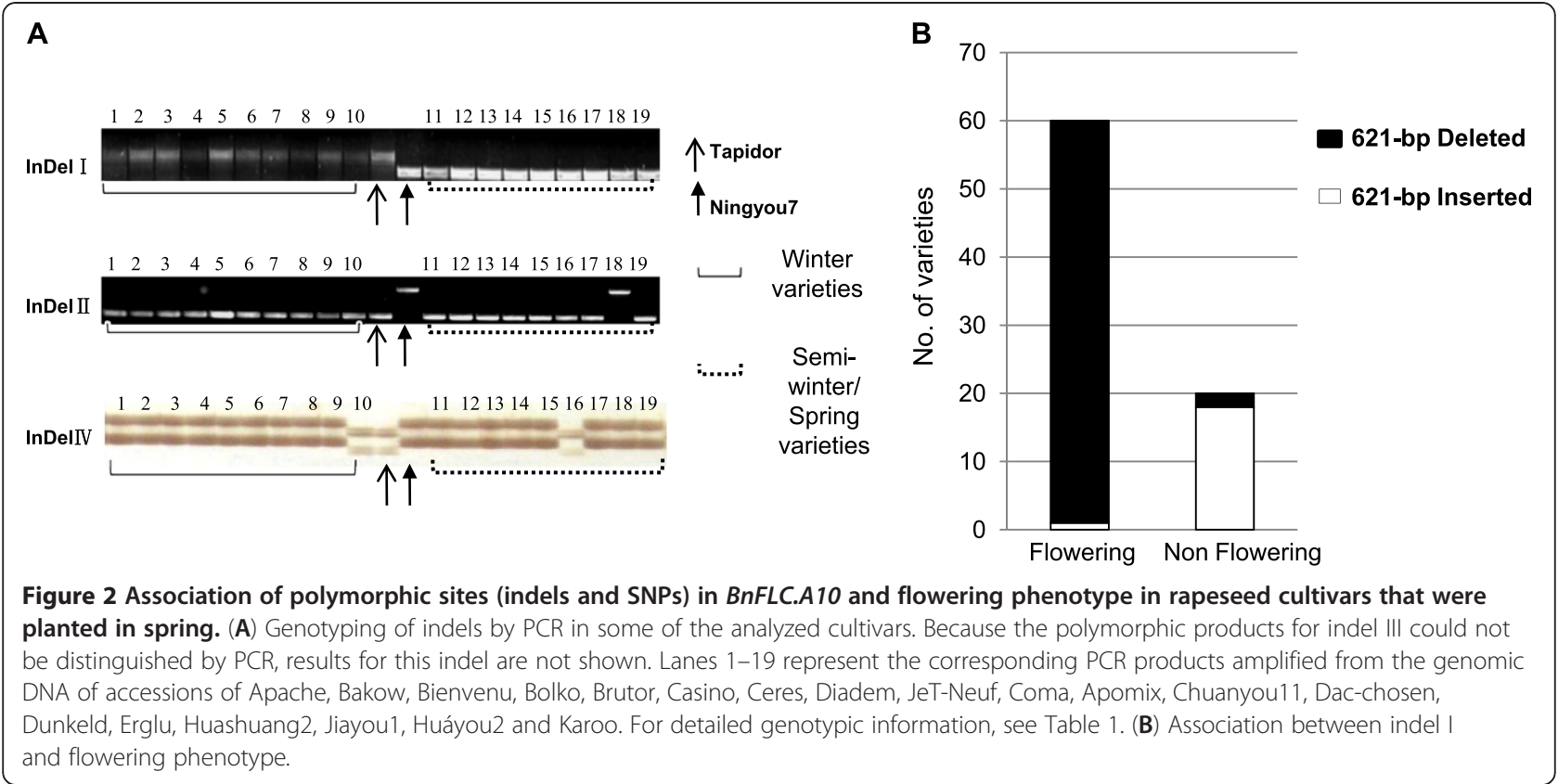

Table 2 Haplotypes detected with the sequence information of BnFLC.A10 from 24 B. napus accessions

\begin{tabular}{|c|c|c|c|c|c|c|c|c|c|c|c|c|c|c|}
\hline \multirow[t]{2}{*}{ Haplotype } & \multirow[t]{2}{*}{ Accession } & \multirow[t]{2}{*}{ Origin } & \multirow[t]{2}{*}{ Type } & \multicolumn{4}{|c|}{ InDel } & \multicolumn{6}{|c|}{ SNP } & \multirow[t]{2}{*}{ Day to flowering } \\
\hline & & & & $1^{* * *}$ & $\|^{*}$ & \|\|$^{\mathrm{a}}$ & $\mathrm{IV}^{*}$ & $1^{*}$ & $2^{*}$ & $3^{*}$ & 4 & 5 & 6 & \\
\hline \multirow[t]{7}{*}{ Hapl } & Tapidor & France & Winter & $\ln$ & DEL & 6 & DEL & G & $\mathrm{T}$ & A & G & G & A & Non-flowering \\
\hline & Apache & UK & Winter & In & DEL & 6 & DEL & G & $\mathrm{T}$ & A & G & G & A & Non-flowering \\
\hline & Bakow & Poland & Winter & In & DEL & 6 & DEL & G & $\mathrm{T}$ & A & G & G & A & Non-flowering \\
\hline & Bienvenu & France & Winter & $\ln$ & DEL & 6 & DEL & G & $\mathrm{T}$ & A & G & G & A & Non-flowering \\
\hline & Brutor & France & Winter & $\ln$ & DEL & 6 & DEL & G & $\mathrm{T}$ & A & G & G & A & Non-flowering \\
\hline & Casino & Sweden & Winter & $\ln$ & DEL & 6 & DEL & G & $\mathrm{T}$ & A & G & G & A & Non-flowering \\
\hline & Quinta & Germany & Winter & In & DEL & 6 & DEL & G & $\mathrm{T}$ & A & G & G & A & Non-flowering \\
\hline \multirow[t]{5}{*}{ Hapll } & Alto & Canada & Spring & DEL & DEL & 6 & DEL & G & $\mathrm{T}$ & A & G & G & A & 62 \\
\hline & Bullet & Canada & Spring & DEL & DEL & 6 & DEL & G & $\mathrm{T}$ & A & G & G & A & 64 \\
\hline & Comet & Denmark & Spring & DEL & DEL & 6 & DEL & G & $\mathrm{T}$ & A & G & G & A & 70 \\
\hline & GULLR & Sweden & Spring & DEL & DEL & 6 & DEL & G & $\mathrm{T}$ & A & G & G & A & 75 \\
\hline & Westar & Canada & Spring & DEL & DEL & 6 & DEL & G & $\mathrm{T}$ & A & G & G & A & 50 \\
\hline Haplll & Qingyou2 & China & Spring & DEL & In & 6 & DEL & G & $\mathrm{T}$ & A & G & G & A & 63 \\
\hline HapIV & Erglu & Australia & Spring & DEL & In & 7 & In & A & C & $\mathrm{T}$ & G & G & A & 69 \\
\hline \multirow[t]{6}{*}{ HapV } & Gānyou5 & China & Semi-winter & DEL & In & 7 & $\ln$ & A & C & $\mathrm{T}$ & A & C & G & 64 \\
\hline & Huashuang1 & China & Semi-winter & DEL & $\ln$ & 7 & In & A & C & $\mathrm{T}$ & A & C & G & 72 \\
\hline & Huāyou4 & China & Semi-winter & DEL & In & 7 & $\ln$ & A & C & $\mathrm{T}$ & A & C & G & 63 \\
\hline & Karoo & Australia & Spring & DEL & In & 7 & In & A & C & $\mathrm{T}$ & A & C & G & 63 \\
\hline & Suyou3 & China & Semi-winter & DEL & In & 7 & In & A & $C$ & $\mathrm{~T}$ & A & C & G & 60 \\
\hline & Xiangyou13 & China & Semi-winter & DEL & In & 7 & In & A & $C$ & $\mathrm{~T}$ & A & C & G & 72 \\
\hline \multirow[t]{4}{*}{ HapVI } & Erra & Germany & Spring & DEL & In & 10 & In & A & $C$ & $\mathrm{~T}$ & A & C & G & 70 \\
\hline & Jiayou1 & China & Spring & DEL & In & 10 & In & A & C & $\mathrm{T}$ & A & C & G & 82 \\
\hline & NingRS-1 & China & Semi-winter & DEL & In & 10 & $\ln$ & A & C & $\mathrm{T}$ & A & C & G & 81 \\
\hline & Ningyou7 & China & Semi-winter & DEL & In & 10 & $\ln$ & A & C & $\mathrm{T}$ & A & C & G & 72 \\
\hline
\end{tabular}

Symbols ${ }^{*}$ and ${ }^{* * *}$ represent $\mathrm{p}=0.05$ and 0.001 levels of significance of each site's influence on flowering time.

aThe repeats of the CTT motif in indel III; In = insertion; $D E L=$ deletion. 


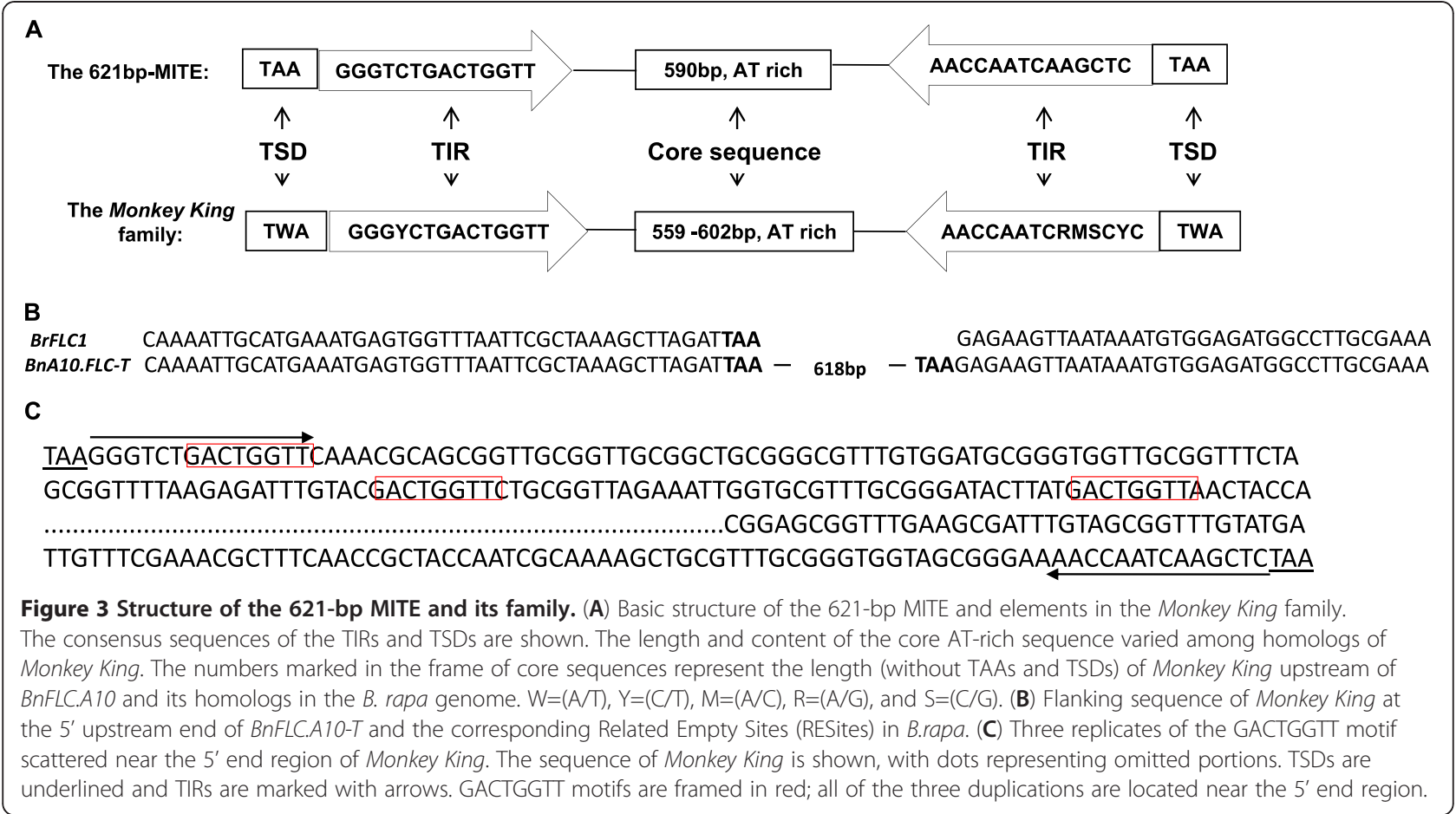

$B n F L C$ copies, or of all BnFLC copies acting in concert in the Coma genome, may be sufficient to inhibit flowering transition under spring environmental conditions, therefore enabling the cultivar 'Coma' to function as a winter rapeseed. Genetic diversity with respect to BnFLCs and other Arabidopsis vernalization pathway gene homologs has been associated with vernalization in B. napus, but no gene or polymorphic site as strongly associated with vernalization requirement of rapeseed as the BnFLC.A10 upstream MITE insertion has been dissected previously. The MITE insertion into BnFLC.A10 appears to be one of the most important causative factors of vernalization requirement in winter rapeseed cultivars.

It is believed that rapeseed originated from a natural hybridization between $B$. rapa and B. oleracea that occurred in southern Europe along the Mediterranean coastline approximately 10,000-100,000 years ago. Given the warm climate in this region year-round, naturally occurring rapeseed genotypes and their ancestors may not have needed to develop an adaptation requiring prolonged vernalization. The activation of Monkey King in B. napus genome would have introduced diversity into the germplasm upon which selective pressure could act. The insertion of Monkey King in the upstream region of BnFLC.A10 resulted in strong dependence on vernalization for flowering; this characteristic was then selected by plant breeders during the development of winter-type rapeseed cultivars of rapeseed that could be grown in northern Europe and other temperate regions of the world.
Several studies have examined the effect of MITEs on neighboring gene expression. For example, the DNA methylation level of a MITE can influence expression of neighboring genes. An assay of transient and stablytransformed rice revealed that the MITE Kiddo, when present in the promoter of the rice ubiquitin2 (rubq2) gene, was responsible for up to $20 \%$ of neighboring gene expression; most notably, when DNA methylation of Kiddo was blocked, transcript levels of endogenous rubq2 increased threefold [44]. An association has also been reported between a MITE inserted in the upstream regulator region of the gene Vgt1 (Vegetative to generative transition) and early flowering in Northern maize genotypes [45,46]. In our study, the MITE upstream of BnFLC.A10 was positively associated with gene expression and induced BnFLC.A10 expression during vernalization. The inserted MITE seems to attenuate cold-induced BnFLC. $A 10$ repression rather than increase its expression, in winter rapeseed. This result is very similar to that observed in Arabidopsis, where FLC expression was correlated with flowering time and vernalization requirement in unvernalized or long days, but not as strongly as anticipated $[47,48]$. We thus conclude that either decreased rate of FLC expression during vernalization or additional epistatic interaction with other genes is more important for control of flowering time and vernalization requirement than variation of $F L C$ expression under unvernalized conditions. Using motif prediction, motifs associated with gene regulation were found to exist in the Monkey 
Table 320 sequences that show high similarity with Monkey King in the B. napus EST library

\begin{tabular}{|c|c|c|c|c|c|}
\hline Accession & $\begin{array}{l}\text { Aligned position in } \\
\text { Monkey King (bp) }\end{array}$ & $\begin{array}{l}\text { Query } \\
\text { coverage }\end{array}$ & E value & $\begin{array}{c}\text { Max } \\
\text { identity }\end{array}$ & Description \\
\hline CD826040.1 & $29-586$ & $90 \%$ & 0 & $89 \%$ & $\begin{array}{l}\text { BN25.062J15F011130 BN25 Brassica napus cDNA clone BN25062J15, } \\
\text { mRNA sequence }\end{array}$ \\
\hline EV022063.1 & $1-416$ & $67 \%$ & 3.00E-164 & $91 \%$ & $\begin{array}{l}\text { BNSCS2CT_UP_086_C03_19APR2007_027 Brassica napus BNSCS2CT } \\
\text { Brassica napus CDNA 5', mRNA sequence }\end{array}$ \\
\hline EE567409.1 & $29-437$ & $66 \%$ & $5.00 \mathrm{E}-157$ & $91 \%$ & $\begin{array}{l}\text { BNZB_UP_149_C07_29SEP2005_059 Brassica napus BNZB Brassica } \\
\text { napus CDNA 5', mRNA sequence }\end{array}$ \\
\hline ES968675.1 & $5-416$ & $66 \%$ & $2.00 \mathrm{E}-151$ & $90 \%$ & $\begin{array}{l}\text { BNZB_UP_208_D10_15MAR2006_074 Brassica napus BNZB Brassica } \\
\text { napus CDNA 5', mRNA sequence }\end{array}$ \\
\hline EE558281.1 & $1-400$ & $64 \%$ & 2.00E-151 & $90 \%$ & $\begin{array}{l}\text { BNZB_RP_027_G10_28APR2004_068 Brassica napus BNZB Brassica } \\
\text { napus CDNA 5', mRNA sequence }\end{array}$ \\
\hline EE564397.1 & $30-613$ & $94 \%$ & $2.00 \mathrm{E}-151$ & $84 \%$ & $\begin{array}{l}\text { BNZB_UP_107_G11_23AUG2004_083 Brassica napus BNZB Brassica } \\
\text { napus CDNA 5', mRNA sequence }\end{array}$ \\
\hline GT085003.1 & $140-613$ & $76 \%$ & 1.00E-143 & $86 \%$ & $\begin{array}{l}\text { c08_20na_1j.s 20na Brassica napus cDNA clone c08_20na_1j 5, mRNA } \\
\text { sequence }\end{array}$ \\
\hline ES956896.1 & $87-416$ & $53 \%$ & $8.00 \mathrm{E}-125$ & $91 \%$ & $\begin{array}{l}\text { 9RDBNGA_UP_157_G11_10MAR2006_083 Brassica napus 9RDBNGA } \\
\text { Brassica napus cDNA 5', mRNA sequence }\end{array}$ \\
\hline EE567134.1 & $379-618$ & $38 \%$ & 4.00E-108 & $96 \%$ & $\begin{array}{l}\text { BNZB_UP_144_H11_27SEP2005_081 Brassica napus BNZB Brassica } \\
\text { napus CDNA 5', mRNA sequence }\end{array}$ \\
\hline EE567253.1 & $373-618$ & $39 \%$ & 1.00E-107 & $95 \%$ & $\begin{array}{l}\text { BNZB_UP_147_A07_27SEP2005_063 Brassica napus BNZB Brassica } \\
\text { napus cDNA 5', mRNA sequence }\end{array}$ \\
\hline EE559708.1 & $379-618$ & $38 \%$ & 1.00E-107 & $96 \%$ & $\begin{array}{l}\text { BNZB_UP_048_D04_11MAY2004_026 Brassica napus BNZB Brassica } \\
\text { napus CDNA 5', mRNA sequence }\end{array}$ \\
\hline EE566332.1 & $315-552$ & $38 \%$ & 2.00E-091 & $92 \%$ & $\begin{array}{l}\text { BNZB_UP_133_A11_27SEP2005_095 Brassica napus BNZB Brassica } \\
\text { napus cDNA 5', mRNA sequence }\end{array}$ \\
\hline ES903789.1 & $5-203$ & $32 \%$ & 3.00E-079 & $93 \%$ & $\begin{array}{l}\text { BNARO4GH_T3_002_A02_24NOV2006_016 Brassica napus BNARO4GH } \\
\text { Brassica napus CDNA 5', mRNA sequence }\end{array}$ \\
\hline EE567417.1 & $390-613$ & $36 \%$ & 9.00E-055 & $84 \%$ & $\begin{array}{l}\text { BNZB_UP_149_D08_29SEP2005_058 Brassica napus BNZB Brassica } \\
\text { napus CDNA 5', mRNA sequence }\end{array}$ \\
\hline FG554276.1 & $441-613$ & $27 \%$ & 7.00E-051 & $88 \%$ & $\begin{array}{l}\text { BN18DYSC_UP_016_A09_18FEB2008_079 BN18DYSC Brassica napus } \\
\text { cDNA 5', mRNA sequence }\end{array}$ \\
\hline EE568964.1 & $438-613$ & $28 \%$ & 1.00E-048 & $86 \%$ & $\begin{array}{l}\text { BNZB_UP_170_G02_30SEP2005_004 Brassica napus BNZB Brassica } \\
\text { napus cDNA 5', mRNA sequence }\end{array}$ \\
\hline EV193796.1 & $438-613$ & $28 \%$ & 1.00E-043 & $85 \%$ & $\begin{array}{l}0091281 \text { Brassica napus Cold acclimation - dark Brassica napus cDNA, } \\
\text { mRNA sequence }\end{array}$ \\
\hline FG577502.1 & $539-613$ & $12 \%$ & 2.00E-021 & $92 \%$ & $\begin{array}{l}\text { BN24DYSC_UP_080_D10_8FEB2008_074 BN24DYSC Brassica napus } \\
\text { cDNA 5', mRNA sequence }\end{array}$ \\
\hline
\end{tabular}

King sequence (Additional file 1). Most of these motifs were located in gene promoter and enhancer regions (TATA box and CAAT box) or were light responsive elements (Sp1) (Additional file 1) associated with response to environmental signals in different organisms. Certain transcriptional factors presumably bind to this region to more efficiently initiate or enhance the expression of neighbouring genes. The actual protein binding ability of the 621-bp insertion was evaluated using electrophoretic mobility shift assays (EMSAs). Nuclear protein(s) extracted from Tapidor before vernalization were able to bind to some fragments from the middle of the 621-bp Monkey King region that contained TATA box motifs (unpublished data). These results suggest that Monkey King can bind to specific transcription factors that may initiate or enhance
BnFLC.A10 expression in winter rapeseed cultivars, giving rise to their stronger vernalization requirement.

Our analysis also indicated that Monkey King is involved in gene regulation in many different settings in the genome. For example, we found three copies of the sequence GACTGGTT near the 5' end of Monkey King (Figure $3 \mathrm{C}$ ); this motif is conserved in the upstream region of Dsg1 (desmoglein1, which encodes desmosomal cadherin) in mice. The motif in Dsg1 is recognized by GRHL1 (grainyhead-like 1, a homolog of the Drosophila gene grainyhead) and increases Dsg1 expression [49]. Part of the Monkey King sequence is transcribed in Brassica genomes (http://www.ncbi.nlm.nih.gov, Table 3), and has been identified in the 3 ' untranslated region of the WRKY21-1 gene (EU912394). Other transcripts that 
Table 4 Sequence information for primers used for polymorphism and BnFLC.A10 gene expression analysis

\begin{tabular}{|c|c|}
\hline Primer name & Sequence(5'-3') \\
\hline \multicolumn{2}{|c|}{ BnFLC.A10 specific primers } \\
\hline Exon1-2 $f$ & CATCCGTCGCTCTTCTTGTC \\
\hline Exon1-2r & GTTGCTTCCATATCGATCAAG \\
\hline Exon2-4 $\mathrm{f}$ & AACATGATGATGATCTTAAAGCCT \\
\hline Exon2-4 $\mathrm{r}$ & CTCCAGCTGAACCAGGGAAC \\
\hline Exon4-7 f & CTTGAGGAATCAAATGTCGATAA \\
\hline Exon4-7 $r$ & CGGAGATTTGTCCTGGTGAG \\
\hline InDell f (P4 f) & GGTCCTTICTITCGTTGGG \\
\hline InDel1 r (P4 r) & GAAGTAAAGTCGGACAAGAAGG \\
\hline InDel2 f (P5 f) & ССTTCTTGTCCGACTTTACTTC \\
\hline InDel2 r (P5 r) & CGTTGCTCCTACTITGTCTATC \\
\hline InDel3 (IP1IP2) f & CGTCGCTCTTCTTGTCGTCTC \\
\hline InDel3 (IP1IP2) r & TATGCATCACAGCGTGTCAAA \\
\hline InDel4 f & GTGTTCAGCTGTCGCTTCTAT \\
\hline InDel4 r & CTAACGCTGGCTTTGATCTT \\
\hline Itrif & AATACTTCCTGCGAATCTTGTG \\
\hline Itr1r & AGTTTGCTTCTAAGTCCCCAAT \\
\hline \multicolumn{2}{|c|}{ SSR primers developed from JBnB75D10 } \\
\hline 25GTTA f & ACTTTCATCACCATTGCAGACA \\
\hline $25 \mathrm{GTTAr}$ & AAGAGCAGCCATTGTATCAGGT \\
\hline T11 f & TTCCCAAGCTTGCTGGTACT \\
\hline T11 r & GAGATTTCCCTCGCTTGATG \\
\hline NIAB009 $\mathrm{f}$ & TACGCTAGTGAGAACACCTCCA \\
\hline NIAB009 $r$ & GCTTAGCAAGAAAACTCGGAA \\
\hline \multicolumn{2}{|l|}{ q-RT PCR primers } \\
\hline Prt $\mathrm{f}$ & TCCGTCGCTCTTCTTGTCGT \\
\hline Prt $r$ & GCTGAACCAGGGAACCCACA \\
\hline $\operatorname{actin} 2 \mathrm{~F}$ & CTGTGCCAATCTACGAGGGTTTC \\
\hline $\operatorname{actin} 2 \mathrm{R}$ & CTTACAATTTCCCGCTCTGCTGT \\
\hline $185 \mathrm{f}$ & GAGTATGGTCGCAAGGCTGAAA \\
\hline $185 \mathrm{r}$ & CGCTCCACCAACTAAGAACGG \\
\hline
\end{tabular}

share high similarity with portions of the Monkey King sequence have been found in the expressed sequence tag library of rapeseed (http://www.ncbi.nlm.nih.gov). The presence of these transcripts suggests the existence of a novel gene regulatory mechanism that is similar to the method by which exon shuffling generates new genes $[50,51]$ or overlapping transcripts generate siRNAs to regulate gene expression $[52,53]$. It is possible that transcripts derived from Monkey King might regulate native gene expression through siRNA-induced DNA methylation. MITE activities within BnFLC.AlO may have shaped phenotypic diversity and influenced mechanisms of adaptation to diverse climates during the evolutionary process.

\section{Conclusions}

This study demonstrated that BnFLC.A10 is the highly likely causative gene underlying $q F T 10-4$, which accounted for most flowering time variation in the TN DH population under spring environmental conditions. Comparision of allelic sequences from Tapidor and Ningyou7 revealed the presence of a Tourist-like MITE insertion in winter-type cultivar Tapidor. Association analysis among winter- and spring-type rapeseeds revealed that the presence of the Tourist-like MITE insertion is very strongly associated with vernalization requirement, and suggested that it appeared after $B$. napus was generated as a product of natural hybridization between B. rapa and B. oleracea. MITE activity led to genetic and phenotypic diversities among varieties and provided the fuel for evolutionary selection. As a result, winter genotypes may have evolved from spring genotypes; this useful variation has subsequently been used as a genetic resource for the development of winter cultivars enabling worldwide production of rapeseed.

\section{Methods}

\section{Plant materials}

For fine mapping of the BnFLC.A10 locus, we used 9,000 plants derived from four $\mathrm{BC}_{5} \mathrm{~F}_{1}$ individuals: 8y0851, 8y086-1, 8y086-2 and 8y086-4. TN DH043 (the 43rd line of the TN DH population) was crossed wiht Ningyou7 (semi-winter recurrent parent) and seeds were collected from the $F_{1}$ generation. Plants were then backcrossed with Ningyou7 over five successive generations $\left(\mathrm{BC}_{1}\right.$ to $\left.\mathrm{BC}_{5}\right)$. Molecular markers were used to track the Tapidor allele at the BnFLC.A10 locus in the F1 backcross. The $\mathrm{BC}_{5} \mathrm{~F}_{2}$ near-isogenic lines were planted in the spring of 2009 for phenotyping with respect to flowering time. A panel of 79 diverse rapeseed cultivars (Table 1) was used for the association analysis. These cultivars were planted in spring during three successive years (20072009) for phenotyping. Climatic conditions during the planting season and geographic features of the planting site were as described previously [36]. The spring rapeseed and $B$. rapa accessions representing nine subspecies that were used to detect the presence of Monkey King upstream of BnFLC.A10 and BrFLC.A10 are listed in Additional file 2. These accessions were obtained from the National Brassica Germplasm Improvement Program (Wagga Wagga, Australia), the Australian Temperate Field Crops Collection (Horsham, Australia), and from the Institute of Vegetables and Flowers, Chinese Academy of Agricultural Sciences (Beijing, China).

\section{Phenotypic evaluation}

Flowering times of the different cultivars used for the association analysis were recorded as the number of days from the day of sowing to the day when $50 \%$ of plants in the plot flowered. In the $\mathrm{BC}_{5} \mathrm{~F}_{2}$ populations, days to 
flowering (DTF) were recorded as the number of days from the day of sowing to the day when the first flower opened. The phenotype 'non-flowering' was assigned when plants showed no visible buds at autumn harvest in middle-October. Phenotypes of the 79 cultivars used for the association analysis are listed in Table 1.

\section{Sequencing of BnFLC.A10 alleles from Tapidor and Ningyou7} A fragment amplified from the Tapidor genome with primer pair "Exon 4-7" (Table 4) was used as a probe to screen the Tapidor BAC library [54]. From 12 BAC clones that contained BnFLC.A10, one clone with the code JBnB75D10 was selected and sequenced to obtain the BnFLC.A10-T allele. Primers (P4, P5, exon1-2,exon2-4, exon4-7, Table 4) were designed based on the basis of the BnFLC.A10-T sequence and used to obtain the sequence of BnFLC.A10-N. The amplicons were cloned into a pGEM-T Easy vector (Promega, Madison, WI, USA) and sequenced to determine the BnFLC.A10-N sequence. Information on primers and amplified gene regions is provided in Table 4. BnFLC.A10$N$ and BnFLC.A10-T sequences were obtained, accession numbers [GenBank: JX901141 and JX901142].

\section{Gene annotation for the BAC sequence}

Gene annotation was carried out using the FGENESH program by selection of the organism category "Dicot plants (A. thaliana)" and alignment with A. thaliana genes. Simple sequences and transposons were identified using RepeatMasker (http://www.repeatmasker.org/, validated 19th September, 2011) followed by manual inspection. We predicted the function of genes that were not aligned with $A$. thaliana orthologs from their conserved domains.

\section{RNA extraction and q-RT PCR}

Plants were grown under long-day conditions $(16 \mathrm{~h}$ light $/ 8 \mathrm{~h}$ dark) at $23^{\circ} \mathrm{C}$ until they had developed to the sixleaf stage, at which point they were transferred to $4^{\circ} \mathrm{C}$ for vernalization. BnFLC.A10 expression was analyzed in plants that had been subjected to a (control), 1, 4 and 7 weeks of vernalization. Total RNA was extracted from plant leaves using TRIzol ${ }^{\circledR}$ reagent (Invitrogen, Carlsbad, California, USA). Total RNA $(2 \mu \mathrm{g})$ was reverse-transcribed using MMLV Reverse Transcriptase (Promega). An iQ5 Real-Time PCR Detection System (Bio-Rad, Hercules, CA, USA) was used for quantitative RT-PCR to detect levels of BnFLC. $A 10$ expression in the two parents. BnFLC.A10 primers (Prt f/Prt r, Table 4) amplified a 235-bp fragment of the BnFLC.A10 CDS. Two genes, actin2 and 18S rRNA (Table 4), were used to normalize expression levels. Three biological and technical replicates were analyzed.

\section{Natural variation in BnFLC.A10}

Allele-specific primers "Itr1f/Itr1r" (Table 4) were used to distinguish the BnFLC.A10 Tapidor allele from the Ningyou7 BnFLC.A10 variant in 24 rapeseed cultivars. The PCR products were cloned into a pGEM-T Easy vector (Promega) for sequencing. Plasmid prepared from two to four colonies from each PCR product was sequenced separately to minimize the contribution of polymerase errors to sequence variation.

\section{Screening of homologous sequences of Monkey King in the $B$. rapa genome}

To identify homologous sequences, the full length MITE sequence was queried against the $B$. rapa genome in the brassicadb database (http://brassicadb.org/) using BLAST. Results were filtered using an $E$ value $<1 \mathrm{e}^{-10}$ as the cutoff.

\section{Additional files}

Additional file 1: Motif prediction of $621 \mathrm{bp}$-MITE.

Additional file 2: Spring rapeseed and $B$. rapa accessions were used for detecting Monkey King existence upstream of BnFLC.A10 and the orthologous region.

Competing interests

The authors declare that they have no competing interests.

\section{Authors' contributions}

$J H, Y L$ and XZ carried out NIL and segregation population development, gene cloning and flowering time investigations; HR and JW conducted phenotypic and association analysis and detected Monkey King in spring rapeseed and B. rapa varieties; SD, QX and $\mathrm{CL}$ conducted flowering time investigations and genotyping of the segregation population; LF performed the MITE structural analysis; BL conducted BAC (JBnB75D10) sequencing; JH and JM designed and supervised the study, analyzed the data and wrote the paper. All the authors discussed the results and contributed to the manuscript. All authors read and approved the final manuscript.

\section{Acknowledgements}

We thank Drs. Caroline Dean (Department of Cell and Developmental Biology, John Innes Centre, UK), Christian Jung (Plant Breeding Institute, Christian-Albrechts-University of Kiel, Germany) and Qifa Zhang (National Key Laboratory of Crop Genetic Improvement, Huazhong Agricultural University, China) for their invaluable comments; Drs Xiaowu Wang (The Institute of Vegetables and Flowers, Chinese Academy of Agricultural Sciences, Beijing, China) and Bob Redden (Horsham) for providing seeds of the Brassica rapa accessions; Rosy Raman and Ms Ekaterina Simova-Samuelian for indel analysis; and Huazhong Agricultural University (Wuhan, China), and NSW DP (Wagga Wagga, Australia) for supporting this research. Financial support for this work was provided by the National Basic Research and Development Programme (2006CB101600) and National Natural Science Foundation of China (30900788)

\section{Author details}

'National Key Laboratory of Crop Genetic Improvement, Huazhong Agricultural University, Wuhan 430070, China. ${ }^{2}$ EH Graham Centre for Agricultural Innovation (an alliance between the Charles Sturt University and NSW Department of Primary Industries), Wagga Wagga Agricultural Institute, Wagga Wagga, NSW 2650, Australia. ${ }^{3}$ Department of Agronomy \& James D. Watson Institute of Genome Sciences, Zhejiang University, Hangzhou 310058, China. ${ }^{4}$ Center of Systematic Genomics, Xinjiang Institute of Ecology and Geography, Chinese Academy of Sciences, Urumqi 830011, China. ${ }^{5}$ Key Laboratory of Experimental Marine Biology, Institute of Oceanology, Chinese Academy of Sciences, Qingdao 266071, China. 
Received: 31 July 2012 Accepted: 30 November 2012

Published: 15 December 2012

\section{References}

1. Primack D, Imbres C, Primack RB, Miller-Rushing AJ, Del Tredici P: Herbarium specimens demonstrate earlier flowering times in response to warming in Boston. Am J Bot 2004, 91(8):1260-1264.

2. Fitter $A H$, Fitter RS: Rapid changes in flowering time in British plants. Science 2002, 296(5573):1689-1691.

3. Mouradov A, Cremer F, Coupland G: Control of flowering time: interacting pathways as a basis for diversity. Plant Cell 2002, 14(Suppl):S111-S130.

4. Simpson GG, Dean C: Arabidopsis, the Rosetta stone of flowering time? Science 2002, 296(5566):285-289.

5. Amasino R: Seasonal and developmental timing of flowering. Plant $J$ 2010, 61(6):1001-1013.

6. Krizek BA, Fletcher JC: Molecular mechanisms of flower development: an armchair guide. Nat Rev Genet 2005, 6(9):688-698.

7. Michaels SD, Amasino RM: FLOWERING LOCUS C encodes a novel MADS domain protein that acts as a repressor of flowering. Plant Cell 1999, 11(5):949-956.

8. Sheldon CC, Burn JE, Perez PP, Metzger J, Edwards JA, Peacock WJ, Dennis ES: The FLF MADS box gene: a repressor of flowering in Arabidopsis regulated by vernalization and methylation. Plant Cell 1999, 11(3):445-458

9. Helliwell CA, Wood CC, Robertson M, James Peacock W, Dennis ES: The Arabidopsis FLC protein interacts directly in vivo with SOC1 and FT chromatin and is part of a high-molecular-weight protein complex. Plant J 2006, 46(2):183-192.

10. Corbesier L, Vincent C, Jang S, Fornara F, Fan Q, Searle I, Giakountis A, Farrona $S$, Gissot L, Turnbull C, et al: FT protein movement contributes to long-distance signaling in floral induction of Arabidopsis. Science 2007 316(5827):1030-1033.

11. Li C, Gu M, Shi N, Zhang H, Yang X, Osman T, Liu Y, Wang H, Vatish M, Jackson S, et al: Mobile FT mRNA contributes to the systemic florigen signalling in floral induction. Sci Rep 2011, 1:73.

12. Kim DH, Doyle MR, Sung S, Amasino RM: Vernalization: winter and the timing of flowering in plants. Annu Rev Cell Dev Biol 2009, 25:277-299.

13. Searle I, He Y, Turck F, Vincent C, Fornara F, Krober S, Amasino RA, Coupland $\mathrm{G}$ : The transcription factor FLC confers a flowering response to vernalization by repressing meristem competence and systemic signaling in Arabidopsis. Genes Dev 2006, 20(7):898-912.

14. Andersson CR, Helliwell CA, Bagnall DJ, Hughes TP, Finnegan EJ, Peacock WJ, Dennis ES: The FLX gene of Arabidopsis is required for FRI-dependent activation of FLC expression. Plant Cell Physiol 2008, 49(2):191-200.

15. Liu F, Quesada V, Crevillen P, Baurle I, Swiezewski S, Dean C: The Arabidopsis RNA-binding protein FCA requires a lysine-specific demethylase 1 homolog to downregulate FLC. Mol Cell 2007, 28(3):398-407.

16. Simpson GG: The autonomous pathway: epigenetic and posttranscriptional gene regulation in the control of Arabidopsis flowering time. Curr Opin Plant Biol 2004, 7(5):570-574.

17. Geraldo N, Baurle I, Kidou S, Hu X, Dean C: FRIGIDA delays flowering in Arabidopsis via a cotranscriptional mechanism involving direct interaction with the nuclear cap-binding complex. Plant Physiol 2009, 150(3):1611-1618.

18. He $Y$, Amasino RM: Role of chromatin modification in flowering-time control. Trends Plant Sci 2005, 10(1):30-35.

19. He Y, Doyle MR, Amasino RM: PAF1-complex-mediated histone methylation of FLOWERING LOCUS C chromatin is required for the vernalization-responsive, winter-annual habit in Arabidopsis. Genes Dev 2004, 18(22):2774-2784.

20. Schmitz RJ, Tamada Y, Doyle MR, Zhang X, Amasino RM: Histone H2B deubiquitination is required for transcriptional activation of FLOWERING LOCUS C and for proper control of flowering in Arabidopsis. Plant Physiol 2009, 149(2):1196-1204.

21. Blanc G, Hokamp K, Wolfe KH: A recent polyploidy superimposed on older large-scale duplications in the Arabidopsis genome. Genome Res 2003, 13(2):137-144.

22. Bowers JE, Chapman BA, Rong J, Paterson AH: Unravelling angiosperm genome evolution by phylogenetic analysis of chromosomal duplication events. Nature 2003, 422(6930):433-438
23. Beilstein MA, Nagalingum NS, Clements MD, Manchester SR, Mathews S: Dated molecular phylogenies indicate a Miocene origin for Arabidopsis thaliana. Proc Natl Acad Sci USA 2010, 107(43):18724-18728.

24. Schranz ME, Lysak MA, Mitchell-Olds T: The ABC's of comparative genomics in the Brassicaceae: building blocks of crucifer genomes. Trends Plant Sci 2006, 11(11):535-542.

25. Yang TJ, Kim JS, Kwon SJ, Lim KB, Choi BS, Kim JA, Jin M, Park JY, Lim MH, Kim HI, et al: Sequence-level analysis of the diploidization process in the triplicated FLOWERING LOCUS C region of Brassica rapa. Plant Cell 2006, 18(6):1339-1347.

26. Naito K, Cho E, Yang G, Campbell MA, Yano K, Okumoto Y, Tanisaka T, Wessler SR: Dramatic amplification of a rice transposable element during recent domestication. Proc Natl Acad Sci USA 2006, 103(47):17620-17625.

27. Jiang N, Bao Z, Zhang X, Hirochika H, Eddy SR, McCouch SR, Wessler SR: An active DNA transposon family in rice. Nature 2003, 421(6919):163-167.

28. Kikuchi K, Terauchi $K$, Wada M, Hirano HY: The plant MITE mPing is mobilized in anther culture. Nature 2003, 421(6919):167-170.

29. Nakazaki T, Okumoto Y, Horibata A, Yamahira S, Teraishi M, Nishida H, Inoue $H$, Tanisaka T: Mobilization of a transposon in the rice genome. Nature 2003, 421(6919):170-172

30. Osborn TC, Kole C, Parkin IA, Sharpe AG, Kuiper M, Lydiate DJ, Trick M: Comparison of flowering time genes in Brassica rapa. B. napus and Arabidopsis thaliana. Genetics 1997, 146(3):1123-1129.

31. Osborn TC, Pires JC, Birchler JA, Auger DL, Chen ZJ, Lee HS, Comai L, Madlung A, Doerge RW, Colot V, et al: Understanding mechanisms of novel gene expression in polyploids. Trends Genet 2003, 19(3):141-147.

32. Putterill J, Robson F, Lee K, Simon R, Coupland G: The CONSTANS gene of Arabidopsis promotes flowering and encodes a protein showing similarities to zinc finger transcription factors. Cell 1995, 80(6):847-857.

33. Wang N, Qian W, Suppanz I, Wei L, Mao B, Long Y, Meng J, Muller AE, Jung C: Flowering time variation in oilseed rape (Brassica napus $L$.) is associated with allelic variation in the FRIGIDA homologue BnaA.FRI.a. J Exp Bot 2011, 62(15):5641-5658.

34. Wang J, Long Y, Wu B, Liu J, Jiang C, Shi L, Zhao J, King GJ, Meng J: The evolution of Brassica napus FLOWERING LOCUS T paralogues in the context of inverted chromosomal duplication blocks. BMC Evol Biol 2009, 9:271.

35. Raman H, Raman R, Eckermann P, Coombes N, Manoli S, Zou X, Edwards D, Meng J, Prangnell R, Stiller J, et al: Genetic and physical mapping of flowering time loci in canola (Brassica napus L.). Theor Appl Genet 2012, doi:10.1007/s00122-012-1966-8.

36. Long Y, Shi J, Qiu D, Li R, Zhang C, Wang J, Hou J, Zhao J, Shi L, Park BS, et al: Flowering time quantitative trait Loci analysis of oilseed brassica in multiple environments and genomewide alignment with Arabidopsis. Genetics 2007, 177(4):2433-2444.

37. Qiu D, Morgan C, Shi J, Long Y, Liu J, Li R, Zhuang X, Wang Y, Tan X Dietrich $E$, et al: A comparative linkage map of oilseed rape and its use for QTL analysis of seed oil and erucic acid content. Theor Appl Genet 2006, 114(1):67-80

38. Bureau TE, Wessler SR: Tourist: a large family of small inverted repeat elements frequently associated with maize genes. Plant Cell 1992, 4(10):1283-1294.

39. Feschotte C, Jiang N, Wessler SR: Plant transposable elements: where genetics meets genomics. Nat Rev Genet 2002, 3(5):329-341.

40. Wessler SR, Bureau TE, White SE: LTR-retrotransposons and MITEs: important players in the evolution of plant genomes. Curr Opin Genet Dev 1995, 5(6):814-821

41. Wang $X$, Wang $H$, Wang J, Sun R, Wu J, Liu S, Bai Y, Mun JH, Bancroft I, Cheng $F$, et al: The genome of the mesopolyploid crop species Brassica rapa. Nat Genet 2011, 43(10):1035-1039.

42. Tadege M, Sheldon CC, Helliwell CA, Stoutjesdijk P, Dennis ES, Peacock WJ: Control of flowering time by FLC orthologues in Brassica napus. Plant $J$ 2001, 28(5):545-553.

43. Zou X, Suppanz I, Raman H, Hou J, Wang J, Long Y, Jung C, Meng J: Comparative Analysis of FLC Homologues in Brassicaceae Provides Insight into Their Role in the Evolution of Oilseed Rape. PLoS One 2012, 7(9):e45751.

44. Yang $G$, Lee $Y$ H, Jiang $Y$, Shi $X$, Kertbundit S, Hall TC: A two-edged role for the transposable element Kiddo in the rice ubiquitin2 promoter. Plant Cell 2005, 17(5):1559-1568.

45. Buckler ES, Holland JB, Bradbury PJ, Acharya CB, Brown PJ, Browne C, Ersoz E, Flint-Garcia S, Garcia A, Glaubitz JC, et al: The genetic architecture of maize flowering time. Science 2009, 325(5941):714-718 
46. Salvi S, Sponza G, Morgante M, Tomes D, Niu X, Fengler KA, Meeley R, Ananiev EV, Svitashev S, Bruggemann E, et al: Conserved noncoding genomic sequences associated with a flowering-time quantitative trait locus in maize. Proc Natl Acad Sci USA 2007, 104(27):11376-11381.

47. Shindo C, Aranzana MJ, Lister C, Baxter C, Nicholls C, Nordborg M, Dean C: Role of FRIGIDA and FLOWERING LOCUS C in determining variation in flowering time of Arabidopsis. Plant Physiol 2005, 138(2):1163-1173.

48. Lempe J, Balasubramanian S, Sureshkumar S, Singh A, Schmid M, Weigel D: Diversity of flowering responses in wild Arabidopsis thaliana strains. PLoS Genet 2005, 1(1):109-118.

49. Wilanowski T, Caddy J, Ting SB, Hislop NR, Cerruti L, Auden A, Zhao LL, Asquith $S$, Ellis $S$, Sinclair R, et al: Perturbed desmosomal cadherin expression in grainy head-like 1-null mice. EMBO J 2008, 27(6):886-897.

50. Patthy L: Genome evolution and the evolution of exon-shuffling-a review. Gene 1999, 238(1):103-114

51. Wang W, Brunet FG, Nevo E, Long M: Origin of sphinx, a young chimeric RNA gene in Drosophila melanogaster. Proc Natl Acad Sci USA 2002, 99(7):4448-4453.

52. Kuang H, Padmanabhan C, Li F, Kamei A, Bhaskar PB, Ouyang S, Jiang J, Buell CR, Baker B: Identification of miniature inverted-repeat transposable elements (MITEs) and biogenesis of their siRNAs in the Solanaceae: new functional implications for MITEs. Genome Res 2009, 19(1):42-56.

53. Vaucheret $\mathrm{H}$ : Post-transcriptional small RNA pathways in plants: mechanisms and regulations. Genes Dev 2006, 20(7):759-771.

54. Rana D, van den Boogaart T, O'Neill CM, Hynes L, Bent E, Macpherson L, Park JY, Lim YP, Bancroft I: Conservation of the microstructure of genome segments in Brassica napus and its diploid relatives. Plant J 2004, 40(5):725-733.

doi:10.1186/1471-2229-12-238

Cite this article as: Hou et al: A Tourist-like MITE insertion in the upstream region of the BnFLC.A10 gene is associated with vernalization requirement in rapeseed (Brassica napus L.). BMC Plant Biology 2012 12:238.

\section{Submit your next manuscript to BioMed Central and take full advantage of:}

- Convenient online submission

- Thorough peer review

- No space constraints or color figure charges

- Immediate publication on acceptance

- Inclusion in PubMed, CAS, Scopus and Google Scholar

- Research which is freely available for redistribution 